\title{
Vibration Attenuation Via Mean of Lower Limb Muscles Occurs During Whole Body Vibrations And Differs Across Frequencies And Postures
}

\section{Isotta Rigoni}

Aston University

Tecla Bonci

University of Sheffield

Paolo Bifulco

University of Naples Federico II

Antonio Fratini ( $\square$ a.fratini@aston.ac.uk)

Aston University

\section{Research Article}

Keywords: whole body vibration (WBV), electromyography (EMG), muscle acceleration, frequency, body posture, tonic vibration reflex

Posted Date: November 15th, 2021

DOI: https://doi.org/10.21203/rs.3.rs-1032225/v1

License: (c) (i) This work is licensed under a Creative Commons Attribution 4.0 International License. Read Full License 


\title{
Vibration attenuation via mean of lower limb muscles occurs during whole body vibrations and differs across frequencies and postures
}

\author{
Isotta Rigoni ${ }^{1,2}$, Tecla Bonci ${ }^{3}$, Paolo Bifulco ${ }^{4}$, Antonio Fratini ${ }^{1^{*}}$
}

1 Biomedical Engineering, College of Engineering and Physical Sciences, Aston University, Birmingham, United Kingdom, 2 EEG and Epilepsy Unit, Clinical Neuroscience Department, University Hospital and Faculty of Medicine of Geneva, Geneva, Switzerland, 3 Department of Mechanical Engineering and the Insigneo Institute for in silico Medicine, University of Sheffield, Sheffield, UK, 4 Department of Electrical Engineering and Information Technology, University of Naples Federico II, Via Claudio 21, 80125, Naples, Italy

*Corresponding author at a.fratini@aston.ac.uk

ORCID:

IR: https://orcid.org/0000-0002-5804-2137

TB: https://orcid.org/0000-0002-8255-4730

PB: https://orcid.org/000-0002-9585-971X

AF: https://orcid.org/0000-0001-8894-461X

Abstract

Lower limb muscles actively contribute to maintain body posture but also act to attenuate soft tissues oscillations that occur during everyday life. This elicited activity can be exploited as a mean of neuromuscular training or rehabilitation. In this study, Whole Body Vibrations (WBV) at different frequencies were delivered to healthy subjects while holding static postures to test the transient muscles mechanical responses. Twenty-five participants underwent WBV at 15, 20, 25 and $30 \mathrm{~Hz}$ while holding either a static 'hack squat' or 'fore feet' posture. Soft tissue accelerations and surface electromyography (sEMG) were recorded from Gastrocnemius Lateralis (GL), Soleus (SOL) and Tibialis Anterior (TA) muscles. Estimated displacement at muscle bellies revealed a resonant pattern, different across frequencies and postures $(p<.001)$. Specifically, a peak in the displacement was measured after the onset of the stimulation, 
1 followed by a drop and a further plateau (only after few seconds after the peak) suggesting a delayed neuromuscular activation. Although oscillation dampening was correlated to an increased muscular activity, only specific WBV settings were promoting a significant muscle contraction. For example, SOL and GL induced activation was maximal for subject in forefeet and while exposed to higher frequencies $(p<.05)$. The non-immediate response of leg muscles to a vibratory stimulation confirms the tonic nature of the vibration induced muscle contraction (the tonic vibration reflex) and its strong influence on postural tonic muscles (GL and SOL). This may have significant impact on training or rehabilitation protocols aiming towards postural and balance improvement or recovery.

Keywords: whole body vibration (WBV); electromyography (EMG); muscle acceleration; frequency; body posture; tonic vibration reflex

\section{Introduction}

Whole Body Vibration (WBV) refers to the use of mechanical stimulation, in the form of vibratory oscillations extended to the whole body, to elicit neuromuscular responses in multiple muscle groups ${ }^{1}$. Vibrations are generally delivered through lower limbs via the use of platforms on which subjects stand. When WBV was included in training and rehabilitation programmes, physical exercises were performed on such platforms ${ }^{2}$. This approach has become increasingly popular as it evokes a large muscle response and, more importantly, it elicits muscles activity through physiological pathways via the Tonic Vibration Reflex (TVR) mechanism ${ }^{3}$, improving the overall motor performance while enhancing strength and flexibility ${ }^{4-9}$.

The TVR has been proven to explain an increased and synchronised motor-unit (MU) firing rates recorded during locally-applied (i.e., focal) vibrations ${ }^{10,11}$. Indeed, when vibrations are applied directly to tendons or muscle bellies, muscle fibres length changes activating a reflex response from muscle spindles. This translates in an increased MU firing rates phased-locked specifically to the vibratory cycle, i.e. the TVR ${ }^{10,12,13}$.

Although in WBV vibrations are not applied locally, they are transferred to the target muscles via the kinematic chain determined by the body posture ${ }^{14-16}$. This provides similar muscular outcomes with respect to focal stimulations as well as additional systemic postural responses, allowing better flexibility and applicability to large exercise programmes ${ }^{4}$. Specifically, when the whole body is exposed to mechanical shocks (such as vibrations), absorption strategies act to dampen oscillations and dissipate energy through modulation of both muscle activity and joint 
1 kinematics, over which the body has prompt control ${ }^{17,18}$. Moreover, in WBV, somatosensory feedback pathways are enhanced by reflexes arising from mechanoreceptors in the lower limbs, with significant implications for motor coordination and postural control during quiet stance ${ }^{19}$.

4 Although promising results of WBV training are reported in the literature ${ }^{20-28}$, a few discording results still jeopardize the systematic use of such approach in training and rehabilitation practices ${ }^{29-31}$. Conflicting results might be related to the high amount of variability in WBV settings (e.g., stimulation frequency, posture, stimulation amplitude, stimulation duration etc.) used throughout different studies, while still lacking of standardised training protocols. Among the most investigated variables, both stimulation frequency and subject posture have relevant impact in eliciting an efficient muscle tuning response to WBV ${ }^{32-34}$. Previous findings suggest that muscles contract to reduce the soft-tissue resonance, especially when the stimulation frequency, $\omega_{a}$, is close to their natural one ${ }^{35-37}$. This process, known as muscle tuning, is perpetrated by muscles to minimize the soft-tissue vibrations ${ }^{38,39}$ and has been recently proposed as one of the possible body reactions to WBV ${ }^{40,41}$. Therefore, a careful selection of stimulation frequency, $\omega_{a}$, to match the resonant one, $\omega_{0}$, seems the key element to maximise muscle responses to WBVs ${ }^{42}$. Generally, the natural frequency of a system depends on its mass, $m$, and stiffness, $k$ according to the formula $\omega_{0}=\sqrt{\mathrm{k} / \mathrm{m}}{ }^{4}$. While the mass of a muscle can be considered as a constant, its stiffness can be modulated by muscle activation in a given body posture. Changes in subjects' posture do therefore change muscles' stiffness, therefore leading to a change in the muscles' natural frequency. During dynamic exercises on a vibrating platform the body kinematic chain involved in the transmission of the mechanical stimulus changes continuously, making it difficult to define the stimulus delivered at the target muscle group. During static WBV exercises instead, the energy dissipated through joint kinematics is constant and muscle contraction is the major mechanism tuned to dampen vibration. Abercromby et al. did in fact confirm that static exercises during WBV enhance muscle response than performing dynamic exercises, during which muscles contract in an eccentric and concentric fashion ${ }^{40}$. However, no actual physiological justification has been provided on the reason why static WBV exercises might be more efficacious than dynamic ones.

We hypothesised that muscles would require an intrinsic time interval to react to the vibratory stimulation (TVR response) and, based on a given stimulus (e.g. frequency) and body posture, to tune muscle stiffness accordingly. In addition, we hypothesised that the extent of vibration dampening is related to the increase of muscle activity and viceversa. To test our hypothesis, we 


\section{$4 \quad$ Materials and Methods}

\section{Participants and experimental design} (reference number: 1439). Italy).

\section{Whole Body Vibration stimulation protocol}

recorded and analysed muscle displacement -derived from accelerometers placed on muscle bellies- and muscle activation in response to WBVs delivered via a side alternating platform at different frequencies while the subjects held different static postures.

Seventeen females and eight males (age: $24.8 \pm 3.4$ years; height: $172.0 \pm 8.6 \mathrm{~cm}$; mass: $64.6 \pm$ $10.5 \mathrm{~kg}$ ) volunteered in the study after providing written informed consent. History of neuromuscular or balance disorders as well as recent injuries were among the exclusion criteria. To evaluate muscle activation and displacement during WBV, surface electromyography (sEMG) signals and accelerations were collected from three lower limb muscles during two static exercises performed in static conditions (without WBV - hereafter called baseline activity) and when different vibration frequencies were delivered. The protocol of the study received approval by the Ethics Committee of the School of Life and Health Sciences at Aston University

Pairs of $\mathrm{Ag} / \mathrm{AgCl}$ surface electrodes (Arbo Solid Gel, KendallTM, CovidienTM $30 \mathrm{~mm} \times 24 \mathrm{~mm}$, centre-to-centre distance $24 \mathrm{~mm}$ ) were placed over the Gastrocnemius Lateralis (GL), Tibialis Anterior (TA) and Soleus (SOL) muscles of the dominant leg, as suggested in the SENIAM guidelines ${ }^{43}$. The reference electrode was placed on the styloid process of the right ulna. The EMG data were sampled at $1000 \mathrm{~Hz}$ (PocketEMG, BTS Bioengineering, Milano, Italy) and sent wirelessly to a laptop via the Myolab software, version 2.12.129.0 (BTS Bioengineering, Milano,

Accelerations were measured via tri-axial accelerometers (AX3, Axivity Ltd, Newcastle, United Kingdom; range $= \pm 16 \mathrm{~g}$, sampling frequency $=1600 \mathrm{~Hz}$ ) placed on $\mathrm{GL}$, TA and SOL muscle bellies, next to the EMG electrodes. The accelerometers were aligned with the $x$-axis parallel to the longitudinal axis of the leg segment, the z-axis normal to the skin surface and the $y$-axis perpendicular to the $x-y$ plane. Accelerations were recorded using the dedicated open source software OMGUI developed by Newcastle University ${ }^{44}$.

Subjects underwent the WBVs barefoot. The WBVs were delivered via a side-alternating platform (Galileo ${ }^{\circ}$ Med, Novotec $\mathrm{GmbH}$, Pforzheim, Germany), as it was shown to evoke bigger 
1 neuromuscular activations than synchronous vibrating ones ${ }^{32}$ : a peak-to-peak amplitude of 4 $\mathrm{mm}$ was used. For each subject, ten trials were collected to evaluate the effect of five stimulation frequencies that covered the frequency range offered by the platform - $0,15,20,25,30 \mathrm{~Hz}$ - and two subject postures: hack squat (HS) and fore-foot (FF). To ensure heels off the ground during the FF trials, subjects were asked to keep their heels in contact with a parallelepiped-shaped foam $(30 \times 4 \times 3 \mathrm{~cm}$ ) glued on the platform while keeping their lower limb straight. During HS trials instead, subjects were asked to keep their knees flexed at about $110^{\circ}$ and a goniometer was used to check the angle at the beginning of each HS trial. Trials were administered in a random order with a one-minute break between consecutive trials.

Hereafter, trials with vibratory stimulation are referred to as the "WBV trials" $\left(H S_{15} H S_{20} H S_{25}\right.$ $H S_{30} F F_{15} F F_{20} F F_{25} F F_{30}$ ) and the others as the "baseline trials" ( $H S_{0}$ and $F F_{0}$ ). WBV trials consisted of 40 seconds: recordings contained 10 seconds with no vibration ( $W B V_{\text {off }}$ portion), once the subject acquired the prescribed posture, followed by 30 seconds of WBVs at the prescribed frequency ( $W B V_{\text {on }}$ portion). Baseline trials were used to assess the relevant subjectspecific EMG baseline activity $\left(H S_{0}\right.$ and $\left.F F_{0}\right)$ over a $30 \mathrm{~s}$ period.

Twelve Vicon Vero v2.2 optical cameras (Vicon Nexus, Vicon Motion Systems Limited, Oxford, UK) were used to measure subjects' posture and assure consistency throughout the experiment. Sixteen retroreflective markers were attached to the participant's body, according to the Plug-In-Gait Lower-Limb model ${ }^{45}$. Data were sampled at $100 \mathrm{~Hz}$ and knee and ankle angles were obtained by extracting the kinematics in the sagittal plane using the proprietary software. Specifically, the ankle and knee angles were used to check for consistency across conditions and subjects.

\section{Data processing and features extraction-Acceleration data}

Raw accelerations from WBV trials were analysed in Matlab ®R2019a (The Mathworks, Inc., Natick, MA). Accelerations were band-pass filtered between 10 and $100 \mathrm{~Hz}$ to remove gravity components and accommodation movements, usually confined between 0 and $5 \mathrm{~Hz}{ }^{46,47}$, and to retain only vibration-induced muscle displacements, located mostly at the stimulation frequency and its superior harmonics ${ }^{48}$. Filtered epochs were then double integrated to estimate local displacement along the different axes $\left(\operatorname{disp}_{x}, \operatorname{disp}_{y}, \operatorname{disp}_{z}\right)$ and the total displacement recorded at each muscle level was estimated as: 


$$
\operatorname{DISP}_{T O T}(\mathrm{t})=\sqrt{\operatorname{disp}_{x}(t)^{2}+\operatorname{disp}_{y}(t)^{2}+\operatorname{disp}_{z}(t)^{2}}
$$

where $t=1,2, \ldots, N$, with $N$ being the total number of samples.

3

4

To track the low-frequency mechanical muscle response to WBVs, a moving average of DISP $_{\text {TOT }}\left(\right.$ MovAvgDISP $_{\text {TOT }}$ ) was calculated using a $250 \mathrm{~ms}$ sliding window (Fig. 1). To compare and superimpose muscle displacement among different subjects, MovAvgDISP $P_{T O T}$ vectors were time-locked to the point where a 0.1 change in the slope was detected, which will be hereafter referred to as the vibration onset, and used for statistical analyses. To describe muscle response to vibrations, two time points were defined as follows:

- $t_{P}$ : time-point in correspondence of the peak, defined as the maximum value of each $M_{\text {ovAvgDISP }}{ }_{\text {TOT }}$ signal in a 2-second interval after the vibration onset (grey area in Fig. 2);

- $t_{A}$ : common attenuation time-point among the different muscles, chosen to represent the response following the peak phase, where muscle displacement is stabilised and minimised, reaching a steady-state. The time-point representing the ending of the peak $\left(t_{\text {EndPeak }}\right)$ was first computed for each muscle and trial: MovAvgDISP ${ }_{T O T}$ signals were averaged across subjects and low-pass filtered at $10 \mathrm{~Hz}$. A $0.5 \mathrm{~s}$ sliding window search was applied to identify the slope change time point $\left(t_{\text {EndPeak }}\right)$, with a threshold of 0.06 . Once a $t_{\text {EndPeak }}$ was identified for all muscles and conditions, $t_{A}$ was defined as:

$$
t_{A}=\operatorname{round}\left(\max \left(t_{\text {EndPeak }}\right)+\frac{1}{2} * \max \left(t_{\text {EndPeak }}\right)\right)
$$

where $\max \left(t_{\text {EndPeak }}\right)$ is the longest peak duration observed across muscles and conditions. Since the longest peak duration was of $3.12 \mathrm{~s}$, which was recorded for the $\mathrm{GL}$ in $H S_{30}, t_{A}$ was located $4.7 \mathrm{~s}$ after the vibration onset (green asterisk in Fig 1).

To quantify the extent of the displacement attenuation at each muscle site, $A T T_{D I S P}$ was calculated for each subject as the difference between the maximum displacement recorded at that site and the steady-state one:

$$
\operatorname{ATT}_{\text {DISP }}=\operatorname{MovAvgDISP_{TOT}}\left(t_{P}\right)-\operatorname{MovAvgDISP} P_{T O T}\left(t_{A}\right)
$$




\section{Data processing and features extraction-EMG Data}

2 To isolate the muscle activity preceding the stimulation $\left(W B V_{o f f}\right)$ from the one actually induced 3 by the vibrations $\left(W B V_{o n}\right)$, each WBV trial was split into two epochs: 10 and 30 seconds, 4 respectively. The central portions of these signals (6 and 20 seconds, respectively) were 5 extracted and retained for analyses. Similarly, the central 20 seconds of the baseline trials $\left(H S_{0}\right.$ 6 and $F F_{0}$ ) were extracted and retained for analyses.

7 All epochs were band-pass filtered between 5 and $450 \mathrm{~Hz}$ with a $5^{\text {th }}$ order Butterworth filter and 8 a mean running root mean square $(r R M S)$ value was obtained from both the baseline $9\left(R M S_{\text {baseline }}\right)$ and the $W B V_{\text {off }}$ epochs $\left(R M S_{W B V o f f}\right)$.

10 To remove motion artefacts from $W B V_{\text {off }}$ and $W B V_{\text {on }}$ epochs ${ }^{48}$, a type II Chebyshev band-stop 11 filter was applied at each stimulation frequency and its harmonics up to $450 \mathrm{~Hz}$ on the EMG 12 spectra. This resulted in 30, 22, 18 ad 15 stop-band filters applied to epochs derived from WBV 13 trials delivered at 15, 20, 25 and $30 \mathrm{~Hz}$, respectively, following the calculation:

$$
\text { \#filters }=\text { round }\left(\frac{\text { frequency spectrum upper limit }}{\text { stimulation frequency }}\right)
$$

For each WBV trial and muscle, two rRMS vectors were computed on both artefact-free epochs $\left(W B V_{o f f} \text { and } W B V_{o n}\right)^{48}$ and used to calculate the relevant mean RMS values: $R M S_{W B V o f f} \sim$ and $R M S_{W B V o n}$, respectively. To compare the values obtained during the different trials, a factor taking into account the proportion of power removed by the comb-notch filter was calculated 49 :

$$
\text { Bias }=\frac{R M S_{W B V o f f \sim}}{R M S_{W B V o f f}}
$$

and was used to adjust $R M S_{W B V o n}$ values:

$$
\operatorname{adjRMS_{WBV}}=R M S_{W B V o n} * \frac{1}{B i a s}
$$
from the $\operatorname{adj} R M S_{W B V}$ obtained for the WBV trials in the respective posture. These resulting values will be hereafter referred to as the increment $R M S_{W B V}$ : 


\section{Statistics}

5 For each muscle, a cluster-based permutation test was used to compare the mechanical

increment $R M S_{W B V}=R M S_{\text {baseline }}-\operatorname{adj} R M S_{W B V}$

In total, eight values were retained for each subject and used for statistical analysis. response of muscles over time ${ }^{50,51}$ for:

- MovAvgDISP $P_{\text {TOT }}$ between the two postures at the four frequencies (four tests);

- MovAvgDISP ${ }_{\text {TOT }}$ between frequency pairs in HS and FF (twelve tests).

Time series comparisons were performed over the portion of the signals between the vibration onset and $t_{A}$ to include both the peak and stabilization phase and because no effect was expected before the WBVs. 5000 permutations were used to build the random distribution against which the test statistic of the actual signal were compared. An alpha level of 0.05 was used to identify the significant clusters for each comparison ${ }^{52}$. To overcome the multiple comparison problem introduced by the number of comparisons run for each muscle, the cluster $p$ values were adjusted with a Bonferroni correction $(p=0.003125)$.

For each muscle, to test whether the electromyography activity increased significantly during the different WBVs, eight Wilcoxon signed rank tests (frequency (4) x posture (2)) compared the incrementRMS $S_{W B V}$ to a normal distribution with zero mean and unknown variance. Analysis were performed in Matlab ®R2019a.

For each analysed muscle, a two-way repeated measures Analysis of Variance (ANOVA) was conducted to examine the effect of the stimulation frequencies and subject postures on incrementRMS $S_{W B V}$ [frequency (4) x posture (2)]. Bonferroni corrections were adopted for multiple comparisons. Since muscle responses were investigated per se, outliers were removed from the dataset of the specific muscle after visual inspection of the data. Residuals were inspected and the approximate normal distribution of the data was confirmed by the AndersonDarling test ${ }^{53}$. Mauchly's test of sphericity was used to assess the sphericity of the data: when the latter was not met, a Greenhouse-Geisser correction was applied. Analysis were run in SPSS 23.0 (IBM Corp., Armonk, NY, USA) ${ }^{54}$. 
To relate the mechanical response with the physiological one, a Pearson correlation coefficient was calculated between $A T T_{D I S P}$ and the outlier-free increment $R M S_{W B V}$ population, after the subjects that were identified as outliers for ANOVA analyses were removed from the respective $A T T_{D I S P}$ population. For each muscle, the data recorded in the eight trials (HS ${ }_{15} H S_{20} H S_{25} H S_{30} F_{15} F_{20} F_{25} F_{30}$ ) were pooled together.

\section{Results}

7 All subjects were able to undergo WBV stimulations while holding the prescribed postures. The 8 average ankle angles measured in Fore Feet were $-9.4^{\circ} \pm 6.4^{\circ}$ where a negative measure 9 indicates a plantar flexion. When participants underwent the WBVs in Hack Squat, the average knee angle was $70.8^{\circ} \pm 4.4^{\circ}$.

\section{Muscle dynamics analysis}

12 Our results confirmed that the muscles dynamics differed significantly depending on the posture and frequency: overall, a larger displacement was observed in HS trials and at lower frequencies.

A characteristic mechanical peak was recorded shortly after the start of the stimulations in both postures, with the only exceptions of TA and SOL muscles when stimulated at $15 \mathrm{~Hz}$. Moreover, although peaks varied among muscles, postures and frequencies, the average displacement showed a similar trend: a peak with a successive drop and a further stabilisation after some seconds (Fig. 2).

More in detail, although peak heights seemed stable across the different explored frequencies, the drop changed significantly among muscles and postures (Fig. 2). GL displacement after the peak, was significantly smaller at higher frequencies - at 20,25 and $30 \mathrm{~Hz}$ rather than at $15 \mathrm{~Hz}$ $(p=.0002)$ and at $30 \mathrm{~Hz}$ rather than at 20 ( $p=.0014)$ (Fig. 2, a.1) in HS. In FF, a similar trend was recorded: a smaller displacement was found at $30 \mathrm{~Hz}$ with respect to $25 \mathrm{~Hz}(\mathrm{p}=.0004)$ (Fig. 2, a.2).

The average displacement recorded at the SOL site was smaller at $30 \mathrm{~Hz}$ than at $15(p=.0006)$, $20(p=.0002)$ and $25 \mathrm{~Hz}(p=.0014)$ while in HS (Fig. 2, b.1). Similarly, in FF, a smaller displacement was recorded at $30 \mathrm{~Hz}$ than at $15(p=.0002), 20(p=.001)$ and $25 \mathrm{~Hz}(p=.0002)$ and at $25 \mathrm{~Hz}$ than at $15 \mathrm{~Hz}(p=.0006)$ (Fig. 2, b.2). 
1 The mechanical response of TA also confirmed the trend observed for the other two muscles:

2 its displacement was always smaller at higher frequencies in $\mathrm{HS}$ ( $H S_{15}<H S_{20}, \mathrm{p}=.0004$;

$3 H S_{25}<H S_{30}, \mathrm{p}=.0006$; for the other comparisons, $\left.\mathrm{p}=.0002\right)$ (Fig. 3, c.1) and in FF ( $\mathrm{p}=.0002$ )

4 (Fig. 3, c.2).

\section{$5 \quad$ Muscle activity analysis}

6 Normality was confirmed for the dependent variables in most of the conditions, for all three 7 muscles. increment $R M S_{W B V}$ was not always normally distributed for TA, but the latter 8 distributions were similarly skewed to those that met normality. Four subjects were removed 9 from the incrementRMS $S_{W B V}$ dataset of $\mathrm{GL}$ and $\mathrm{SOL}$, and three from that of $\mathrm{TA}$, since 10 represented outlier values. Distribution of increment $R M S_{W B V}$ values for the different muscles, 11 posture and frequencies is depicted in Fig. 3.

A significant WBV-induced muscle activation (increment $R M S_{W B V}$ ) was observed in all conditions for the GL (see first row of Table 1) and in most of the conditions for the SOL, apart from $H S_{15}$ (see second row of Table 1). Instead, the TA showed a significant response to WBVs only for 15 and $30 \mathrm{~Hz}$ and $F F_{25}$ (see third row of Table 1 ).

ANOVA analyses showed that although no significant interaction was found for the $G L(N=21)$, main effect of stimulation frequency $(F(3,60)=14.397, p<.0001)$ and subject posture were statistically significant $(F(1,20)=15.433, p=.001)$. Specifically, GL-sEMG activity increased more in FF than in $\mathrm{HS}(p=.001)$ and $30 \mathrm{~Hz}$ was the stimulation frequency that evoked the highest muscular activation when compared to $15 \mathrm{~Hz}(p<.0001)$ and $20 \mathrm{~Hz}(p=.001)$. The WBV-induced increment of GL activation was also higher at $25 \mathrm{~Hz}$ than at $20 \mathrm{~Hz}(p=.02)$. Similarly, no significant interaction was found for the SOL $(\mathrm{N}=21)$ and a similar stimulation frequency $(F(1.772,35.434)$ $=12.982, \mathrm{p}<.0001))$ and subject posture $(F(1,20)=6.357, \mathrm{p}=.02))$ main effects were found. The WBV-induced increment of SOL activity was higher in FF than in $\mathrm{HS}(p=.02)$ and $30 \mathrm{~Hz}$ was the stimulation frequency in which the highest SEMG increment was found when compared to 15 $\mathrm{Hz}(p=.002), 20 \mathrm{~Hz}(p=.004)$ and $25 \mathrm{~Hz}(p=.037)$. Moreover, a $25 \mathrm{~Hz}$ stimulation led to a higher muscle activation than $20 \mathrm{~Hz}(p=.009)$. No significant interaction nor main effect was instead found for the TA $(\mathrm{N}=22)$. 


\section{Relation between muscle dynamics and muscle activity}

2 A positive correlation was found between the increase of SOL muscle activity and the amount of displacement attenuation ( $r h o=0.2886, p<.001$, see Fig. 4 , B). No significant correlations were found between the augmented activation of GL and TA and the extent of displacement reduction measured at the respective site (Fig. 4, A and C).

\section{Discussions}

7 To the authors knowledge, this is the first study analysing the dynamics of the mechanical response of muscle tissues to WBVs and to correlate it with EMG activity to highlight an immediate or delayed response to steady stimulations. Some studies have related the platform acceleration to muscle activity 55,56 , others investigated the relationship between muscle activation and body joint acceleration ${ }^{34,57,58}$ and others studied the transmissibility of vibrations to the shank and thigh segments in relation to muscle activity ${ }^{59,60}$. In the latter, a single body posture was used, and the analyses focussed only on the central part of the WBV trials, leaving out the analyses of the initial response to the stimulation. No other study was found to analyse the progressive dynamics of the displacement at the muscle site and EMG activation while undergoing WBVs with different static postures.

Our analysis of the dynamics of the displacement and EMG recorded at each muscle site confirmed our hypotheses: muscle reaction to WBVs depends on stimulus characteristics and subject's posture and develops in time to reduce muscle oscillations. Indeed, a common mechanical pattern, never highlighted before, can be observed from our results (Fig. 3). In response to vibratory stimulations, the extent of oscillations of muscles shows a rising phase, a peak oscillation and a subsequent drop, all of which completed within 4 to 5 seconds after the vibration onset, followed by a sustained stable oscillation (plateau). Neither the stimulation amplitude nor the posture of participants varied during individual tests, hence a neuromuscular response is accounted for the observed dynamics. This interpretation aligns to the muscle tuning theory, whereby soft-tissue oscillations arising in response of impact forces applied to the feet are dampened by an increase in muscle activation ${ }^{37,38,61}$. During WBVs, in fact, vibrations are transferred from the feet to the muscles via the body kinematic chain and produce soft-tissue compartment oscillations at the stimulation frequency, which in our case was in the range of the natural frequencies of calf muscles ${ }^{35}$. In light of the reported theory, it is therefore reasonable to assume that, if a resulting potential resonance is detected, muscle contraction is increased to 
avoid damage, creating the characteristic raising and falling curves observed in our recordings. The differences observable in these curves confirm that mechanical response changes across muscles, frequencies and posture, suggesting that it is not of artefactual nature but that it actually reflects an underlying muscular activation. Moreover, they also suggest that not all combinations of frequencies and postures can elicit a resonant response in some muscles. A resonant peak is in fact completely absent in the response of SOL muscle at $15 \mathrm{~Hz}$ (in both FF and $\mathrm{HS}$ ) and at $20 \mathrm{~Hz}$ in HS. Similarly, no peak is observable in the TA response to $15 \mathrm{~Hz}$ WBVs delivered in HS. These results resemble those obtained by Pollock et al (2010), where $15 \mathrm{~Hz}$ represented the upper limit for transmissibility of vibrations from the platform. The accelerations at the knee joint were found to peak at $15 \mathrm{~Hz}$ and to dramatically decrease with increasing frequencies, suggesting the occurrence of muscle tuning ${ }^{34}$. Similarly, vibration transmission to the triceps surae and thigh muscle compartments were found to consistently decrease with increasing frequencies ${ }^{59}$, suggesting that a damping effect was more present at frequencies that are closer to the muscles' resonant ones (the higher ones). These results are in line with what we observe in the plateau phase of the mechanical response, nevertheless, these conclusions were drawn on partial information analyses (the central interval of the WBV trials), and do not include the analysis of the initial dynamics.

Our study advance the understanding of muscles reaction to WBVs according to stimulation characteristics and, specifically, highlight the tonic nature of muscle reaction to vibration. Indeed, only after an intrinsic interval, which in this study is around 5 seconds, this reaction can completely settle. This may also explain why static exercises (postures) are found to be more effective than the dynamic ones while on vibrating platforms ${ }^{40}$ : during the first, muscles can tune to WBVs as opposed to a continuously changing kinematic chain, with changing in muscle contraction and sensitivity to vibrations ${ }^{11}$.

In addition, the analysis of the physiological response of muscles to WBVs highlighted specific combinations of posture/frequency able to produce maximal results. As expected from acceleration analyses, also muscles activation varied: GL SEMG activity was significantly enhanced in all WBV combinations, while only specific combinations were effective for SOL and TA activation. This highlight the importance of the selection of appropriate WBV parameters combinations to activate target muscles. In addition, undergoing WBV stimulation while in Fore Feet was found to lead to a higher increase of GL and SOL SEMG activity rather than in Hack Squat position, confirming previous research findings ${ }^{32}$. Contracted muscles are in fact more 
1 responsive ${ }^{11}$, and in our case $\mathrm{GL}$ and $\mathrm{SOL}$, both plantar-flexors, are more engaged in FF than $\mathrm{HS}$ 2 $62,63$.

3 WBVs delivered at 30 and $25 \mathrm{~Hz}$ triggered a greater activation in both muscles, as similar findings reported ${ }^{64}$, supporting previous proposal of GL natural frequency residing between 25 and 30 $\mathrm{Hz}{ }^{42}$. These conclusions are further confirmed by the observation of the permutation test results. Most differences were appreciable for the plateau phase, where the displacement of GL and SOL soft-tissue compartments was significantly reduced at $30 \mathrm{~Hz}$ than at other WBV frequencies, further supporting the claim that this frequency is the one triggering the largest tuning effect. Moreover, the positive correlation found between the SOL SEMG increase and the displacement attenuation further suggest that the reduction of displacement in the plateau phase is indeed the manifestation of a neuromuscular response, potentially activated to reduce resonance. The absence of correlation in GL and TA might be explained by the sub-population separation visible in the first and the absence of variance in one of the two population visible in the latter.

The absence of any posture or frequency effect on the TA activation during WBVs might be explained by the following: (i) the stimulation frequencies used in this study that were limited to $30 \mathrm{~Hz}$ and not enough close to TA's natural frequency, which ranges up to $50 \mathrm{~Hz}{ }^{36}$; (ii) the selected postures that did not lead to an appropriate level of TA engagement, limiting its response to WBVs ${ }^{11}$; (iii) the phasic nature of the TA, which makes it physiologically different from the other muscles included in this study ${ }^{65}$.

Combining the above, it can be inferred that $30 \mathrm{~Hz}$-Fore Feet might be the best combination of stimulation frequency and subject posture when aiming to effectively enhance both GL and SOL muscular responses. For the explored combinations, instead, the TA muscle showed that WBVs elicit muscular activity but did not allow to identify any combination producing a significantly higher response. Therefore, a wider range of frequencies and postures or a completely different approach should be explored.

For further studies on the topic, synchronisation of EMG recordings, soft tissue and platform accelerations should be carefully considered and justified. Vibration propagation does in fact depends not only on the level of stiffness of muscles, but also on the gender and anthropometrics of the subjects ${ }^{66}$. With the procedure adopted in this study, it was possible to align the soft-tissue accelerations/EMG recordings at the time where the tissue begins to 


\section{Conclusions}

7 Our results highlighted a muscle driven mechanical response in muscles undergoing vibratory 8 stimulation: a clear trend with a resonant peak followed, after few seconds from the start of the 9 stimulus, by a more stable plateau that reflects a "delayed" neuromuscular activation to modify

oscillate (rather than on the platform onset). This allowed a more appropriate synchronisation of muscle activity and mechanical response between subjects.

In addition, although the WBV frequencies investigated in our study encompass the range commonly used in WBV training ${ }^{59}$, future studies should expand the investigation to higher frequencies.

$$
\text { the properties of the biomechanical system (e.g. muscle stiffness). The non-immediate response }
$$
of leg muscles to a vibratory stimulation confirms the tonic nature of vibration-induced muscle contraction and its strong influence on postural tonic muscles (GL and SOL). Furthermore, EMG analyses suggest that calf muscles produce maximal response if participants are standing on the fore feet during stimulations in a range of $25-30 \mathrm{~Hz}$. Our results suggest that to elicit a stable tonic muscle contraction while using a vibrating platform, training programmes should consider only static postures, or in alternative, participants should be instructed to hold the same posture for longer than five seconds. This approach therefore may have profound impact on training or rehabilitation protocols aiming towards postural and balance improvement or recovery.

\section{References}

1. Cardinale, M. \& Wakeling, J. Whole body vibration exercise: Are vibrations good for you? Br. J. Sports Med. 39, 585-589 (2005).

2. Dolny, D. G. \& Reyes, F. C. G. Whole body vibration exercise: Training and benefits. Curr. Sports Med. Rep. 7, 152-157 (2008).

3. Granit, R. \& Steg, G. Tonic and Phasic Ventral Horn Cells Differentiated by Post-Tetanic Potentiation in Cat Extensors. Acta Physiol Scand 37, 114-126 (1956).

4. Rittweger, J. Vibration as an exercise modality: How it may work, and what its potential might be. Eur. J. Appl. Physiol. 108, 877-904 (2010).

5. Delecluse, C., Roelants, M. \& Verschueren, S. Strength increase after whole-body vibration compared with resistance training. Med. Sci. Sports Exerc. 35, 1033-1041 (2003).

6. Wyon, M., Guinan, D. \& Hawkey, A. Whole-Body vibration training increases vertical jump height in a dance population. J. Strength Cond. Res. 24, 866-870 (2010). 
7. Osawa, Y., Oguma, Y. \& Ishii, N. The effects of whole-body vibration on muscle strength and power: A meta-analysis. J. Musculoskelet. Neuronal Interact. 13, 342-352 (2013).

8. Alam, M. M., Khan, A. A. \& Farooq, M. Effect of whole-body vibration on neuromuscular performance: A literature review. Work 59, 571-583 (2018).

9. Saquetto, M., Carvalho, V., Silva, C., Conceição, C. \& Gomes-Neto, M. The effects of whole body vibration on mobility and balance in children with cerebral palsy: A systematic review with metaanalysis. J. Musculoskelet. Neuronal Interact. 15, 137-144 (2015).

10. Burke, D. \& Schiller, H. H. Discharge pattern of single motor units in the tonic vibration reflex of human triceps surae. J. Neurol. Neurosurg. Psychiatry 39, 729-741 (1976).

11. Burke, D., Hagbarth, K. E., Lofstedt, L. \& Wallin, B. G. The responses of human muscle spindle endings to vibration during isometric contraction. J. Physiol. 261, 695-711 (1976).

12. Hagbarth, K. E., Hellsing, G. \& Löfstedt, L. TVR and vibration-induced timing of motor impulses in the human jaw elevator muscles. J. Neurol. Neurosurg. Psychiatry 39, 719-728 (1976).

13. Person, R. \& Kozhina, G. Tonic vibration reflex of human limb muscles: Discharge pattern of motor units. J. Electromyogr. Kinesiol. 2, 1-9 (1992).

14. Ritzmann, R., Kramer, A., Gruber, M., Gollhofer, A. \& Taube, W. EMG activity during whole body vibration: Motion artifacts or stretch reflexes? Eur. J. Appl. Physiol. 110, 143-151 (2010).

15. Pollock, R. D., Woledge, R. C., Martin, F. C. \& Newham, D. J. Effects of whole body vibration on motor unit recruitment and threshold. J. Appl. Physiol. 112, 388-395 (2012).

16. Cardinale, M. \& Lim, J. Electromyography activity of vastus lateralis muscle during whole-body vibrations of different frequencies. J. Strength Cond. Res. 17, 621-624 (2003).

17. Gross, T. S. \& Nelson, R. C. The shock attenuation role of the ankle during landing from a vertical jump. Med. Sci. Sports Exerc. 20, 506-514 (1988).

18. Lafortune, M. A., Lake, M. J. \& Hennig, E. M. Differential shock transmission response of the human body to impact severity and lower limb posture. J. Biomech. 29, 1531-1537 (1996).

19. Fitzpatrick, R. C., Gorman, R. B., Burke, D. \& Gandevia, S. C. Postural proprioceptive reflexes in standing human subjects: bandwidth of response and transmission characteristics. J. Physiol. 458, 69-83 (1992).

20. Lam, F. M. H., Lau, R. W. K., Chung, R. C. K. \& Pang, M. Y. C. The effect of whole body vibration on balance, mobility and falls in older adults: A systematic review and meta-analysis. Maturitas 72, 206-213 (2012).

21. Ritzmann, R., Kramer, A., Bernhardt, S. \& Gollhofer, A. Whole body vibration training - Improving balance control and muscle endurance. PLoS One 9, (2014).

22. Bautmans, I., Van Hees, E., Lemper, J. C. \& Mets, T. The feasibility of whole body vibration in institutionalised elderly persons and its influence on muscle performance, balance and mobility: A randomised controlled trial [ISRCTN62535013]. BMC Geriatr. 5, 1-8 (2005).

23. Torvinen, S. et al. Effect of a vibration exposure on muscular performance and body balance. Randomized cross-over study. Clin. Physiol. Funct. Imaging 22, 145-152 (2002).

24. Mahieu, N. N. et al. Improving strength and postural control in young skiers: Whole-body vibration versus equivalent resistance training. J. Athl. Train. 41, 286-293 (2006).

25. Rogan, S., Hilfiker, R., Herren, K., Radlinger, L. \& De Bruin, E. D. Effects of whole-body vibration on postural control in elderly: A systematic review and meta-analysis. BMC Geriatr. 11, (2011).

26. Bogaerts, A., Verschueren, S., Delecluse, C., Claessens, A. L. \& Boonen, S. Effects of whole body vibration training on postural control in older individuals: A 1 year randomized controlled trial. Gait Posture 26, 309-316 (2007).

27. van Nes, I. ;, Geurts, A. ;, Hendricks, H. T. . \& Duysens, J. Short-Term Effects of Whole-Body 
Vibration on Postural Control in Unilateral Chronic Stroke Patients: Preliminary Evidence. Am. J. Phys. Med. Rehabil. 83, 867-873 (2004).

28. Turbanski, S., Haas, C. T., Schmidtbleicher, D., Friedrich, A. \& Duisberg, P. Effects of random whole-body vibration on postural control in Parkinson's disease. Res. Sport. Med. 13, 243-256 (2005).

29. Yang, X., Wang, P., Liu, C., He, C. \& Reinhardt, J. D. The effect of whole body vibration on balance, gait performance and mobility in people with stroke: A systematic review and metaanalysis. Clin. Rehabil. 29, 627-638 (2015).

30. Torvinen, S. et al. Effect of 4-min vertical whole body vibration on muscle performance and body balance: A randomized cross-over study. Int. J. Sports Med. 23, 374-379 (2002).

31. Torvinen, S. et al. Effect of 8-month vertical whole body vibration on bone, muscle performance, and body balance: a randomized controlled study. J. Bone Miner. Res. 18, 876-884 (2003).

32. Ritzmann, R., Gollhofer, A. \& Kramer, A. The influence of vibration type, frequency, body position and additional load on the neuromuscular activity during whole body vibration. Eur. J. Appl. Physiol. 113, 1-11 (2013).

33. Lienhard, K., Cabasson, A., Meste, O. \& Colson, S. S. Determination of the optimal parameters maximizing muscle activity of the lower limbs during vertical synchronous whole-body vibration. Eur. J. Appl. Physiol. 114, 1493-1501 (2014).

34. Pollock, R. D., Woledge, R. C., Mills, K. R., Martin, F. C. \& Newham, D. J. Muscle activity and acceleration during whole body vibration: Effect of frequency and amplitude. Clin. Biomech. 25, 840-846 (2010).

35. Wakeling, J. \& Nigg, B. Modification of soft tissue vibrations in the leg by muscular activity. J Appl Physiol 90, 412-420 (2001).

36. Wakeling, J., Nigg, B. \& Rozitis, A. Muscle activity damps the soft tissue resonance that occurs in response to pulsed and continuous vibrations. J. Appl. Physiol. 93, 1093-1103 (2002).

37. Wakeling, J., Liphardt, A. M. \& Nigg, B. Muscle activity reduces soft-tissue resonance at heelstrike during walking. J. Biomech. 36, 1761-1769 (2003).

38. Wakeling, J. \& Nigg, B. Soft-tissue vibrations in the quadriceps measured with skin mounted transducers. J. Biomech. 34, 539-543 (2001).

39. Wakeling, J., Von Tscharner, V., Nigg, B. \& Stergiou, P. Muscle activity in the leg is tuned in response to ground reaction forces. J. Appl. Physiol. 91, 1307-1317 (2001).

40. Abercromby, A. et al. Variation in neuromuscular responses during acute whole-body vibration exercise. Med. Sci. Sports Exerc. 39, 1642-1650 (2007).

41. Abercromby, A. et al. Vibration exposure and biodynamic responses during whole-body vibration training. Med. Sci. Sports Exerc. 39, 1794-1800 (2007).

42. Cesarelli, M. et al. Analysis and modelling of muscles motion during whole body vibration. EURASIP J. Adv. Signal Process. 2010, 26-28 (2010).

43. Hermens, H. J., Bart, F., Catherine, D.-K. \& Gunter, R. Development of recommendations for SEMG sensors and sensor placement procedures. J. Electromyogr. Kinesiol. 10, 361-374 (2000).

44. AX3 OMGUI Configuration and Analysis Tool, v38, GitHub. (2015).

45. Davis III, R. B., Ounpuu, S., Tyburski, D. \& Gage, J. R. A gait analysis data collection and recution technique. Hum. Mov. Sci. 10, 575-587 (1991).

46. Nowak, D. A., Rosenkranz, K., Hermsdörfer, J. \& Rothwell, J. Memory for fingertip forces: Passive hand muscle vibration interferes with predictive grip force scaling. Exp. Brain Res. 156, 444-450 (2004).

47. Prieto, T. E., Myklebust, J. B., Hoffmann, R. G., Lovett, E. G. \& Myklebust, B. M. Measures of 
postural steadiness: Differences between healthy young and elderly adults. IEEE Trans. Biomed. Eng. 43, 956-966 (1996).

48. Fratini, A., Cesarelli, M., Bifulco, P. \& Romano, M. Relevance of motion artifact in electromyography recordings during vibration treatment. J. Electromyogr. Kinesiol. 19, 710-718 (2009).

49. Lienhard, K., Cabasson, A., Meste, O. \& Colson, S. S. Comparison of sEMG processing methods during whole-body vibration exercise. J. Electromyogr. Kinesiol. 25, 833-840 (2015).

50. Maris, E. \& Oostenveld, R. Nonparametric statistical testing of EEG-and MEG-data. J. Neurosci. Methods 164, 177-190 (2007).

51. Maris, E. Statistical testing in electrophysiological studies. Psychophysiology 49, 549-565 (2012).

52. Gerber, E. M. permutest. (2020). Available at: https://uk.mathworks.com/matlabcentral/fileexchange/71737-permutest. (Accessed: 20th May 2020)

53. Mohd Razali, N. \& Bee Wah, Y. Power comparisons of Shapiro-Wilk, Kolmogorov-Smirnov, Lilliefors and Anderson-Darling tests. J. Stat. Model. Anal. 2, 21-33 (2011).

54. Field, A. Discovering Statistics using IBM SPSS statistics. (SAGE Publication Ltd, 2013).

55. Lienhard, K. et al. Relationship Between Lower Limb Muscle Activity and Platform Acceleration During Whole-Body Vibration Exercise. J. Strength Cond. Res. 29, 2844-53 (2015).

56. Di Giminiani, R., Masedu, F., Padulo, J., Tihanyi, J. \& Valenti, M. The EMG activity-acceleration relationship to quantify the optimal vibration load when applying synchronous whole-body vibration. J. Electromyogr. Kinesiol. 25, 853-859 (2015).

57. Tankisheva, E. et al. Transmission of whole body vibration and its effect on muscle activation. J. Strength Cond. Res. 27, 2533-2541 (2013).

58. Beerse, M., Lelko, M. \& Wu, J. Acute effect of whole-body vibration on acceleration transmission and jumping performance in children. Clin. Biomech. 81, 105235 (2021).

59. Friesenbichler, B., Lienhard, K., Vienneau, J. \& Nigg, B. M. Vibration transmission to lower extremity soft tissues during whole-body vibration. J. Biomech. 47, 2858-2862 (2014).

60. Cook, D. P. et al. Triaxial modulation of the acceleration induced in the lower extremity during whole-body vibration training: a pilot study. J. Strength Cond. Res. 298-308 (2011).

61. Wakeling, J., Pascual, S. \& Nigg, B. Altering muscle activity in the lower extremities by running with different shoes. Med. Sci. Sport. Exerc. 34, 1529-1532 (2002).

62. Okada, M. An electromyographic estimation of the relative muscular load in different human postures. J. Hum. Ergol. (Tokyo). 1, 75-93 (1972).

63. Carlsöö, S. The static muscle load in different work positions: An electromyographic study. Ergonomics 4, 193-211 (1961).

64. Di Giminiani, R., Masedu, F., Tihanyi, J., Scrimaglio, R. \& Valenti, M. The interaction between body position and vibration frequency on acute response to whole body vibration. $J$. Electromyogr. Kinesiol. 23, 245-251 (2013).

65. Honma, S. \& Seki, Y. Muscle spindles in phasic and tonic muscle. Tohoku J. Exp. Med. 83, 391397 (1964).

66. Dewangan, K. N., Shahmir, A., Rakheja, S. \& Marcotte, P. Vertical and fore-aft seat-to-head transmissibility response to vertical whole body vibration: Gender and anthropometric effects. J. Low Freq. Noise Vib. Act. Control 32, 11-40 (2013).

67. Morel, P. Gramm: grammar of graphics plotting in Matlab. J. Open Source Softw. 3, 568 (2018). 


\section{Author's contributions}

2 Conception and design: I. R., T. B., P. B. and A. F. Data acquisition and analysis: I. R.

3 Interpretation: I. R. and A. F. Drafting manuscript: I. R., T. B., P.B. and A. F.

\section{Additional Information}

5 Competing interests All authors declare that they have no conflicts of interest.

6 Availability of data and material Since sharing data in an open-access repository was not

7 included in our participant's consent and therefore compromises our ethical standards, data are

8 only available on request from the corresponding author.

9 Code availability The code used for the analyses of the data will be shared upon request to the 10 corresponding author

11 Ethics approval The study was carried out according to the Declaration of Helsinki (2013) and

12 was approved by the University Research Ethics Committee at Aston University (reference 13 number: 1439).

14 Consent to participate All participants provided informed consent before participating.

15 Consent for publication All co-authors were aware of the publication of this study 


\section{Figures}
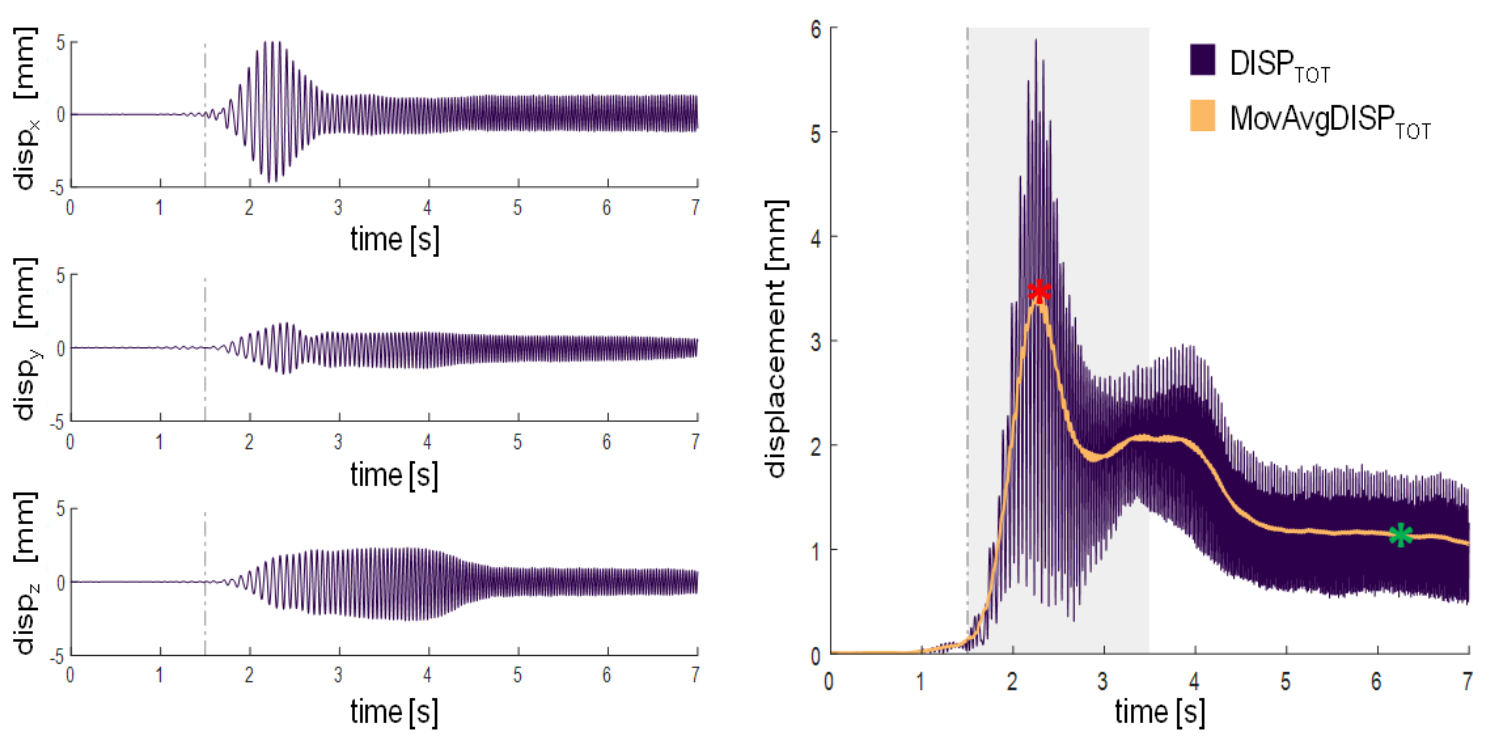

Figure 1: Extraction of muscle dynamics. On the left panel, muscle displacement obtained from double integration of the soft-tissue acceleration recorded at the $G L$ site, $H S_{30}$. Displacement along time is reported for the $x, y$ and $z$ axis. On the right panel, the GL total displacement obtained from the combination of the signals on the left (in purple): the moving average is depicted in orange. The vibration onset is indicated on the graphs by the vertical dashed line; the two-second interval used for the search of $t_{P}$ is highlighted with a grey area- The red and green asterisk indicate $t_{P}$ and $t_{A}$ respectively.

9
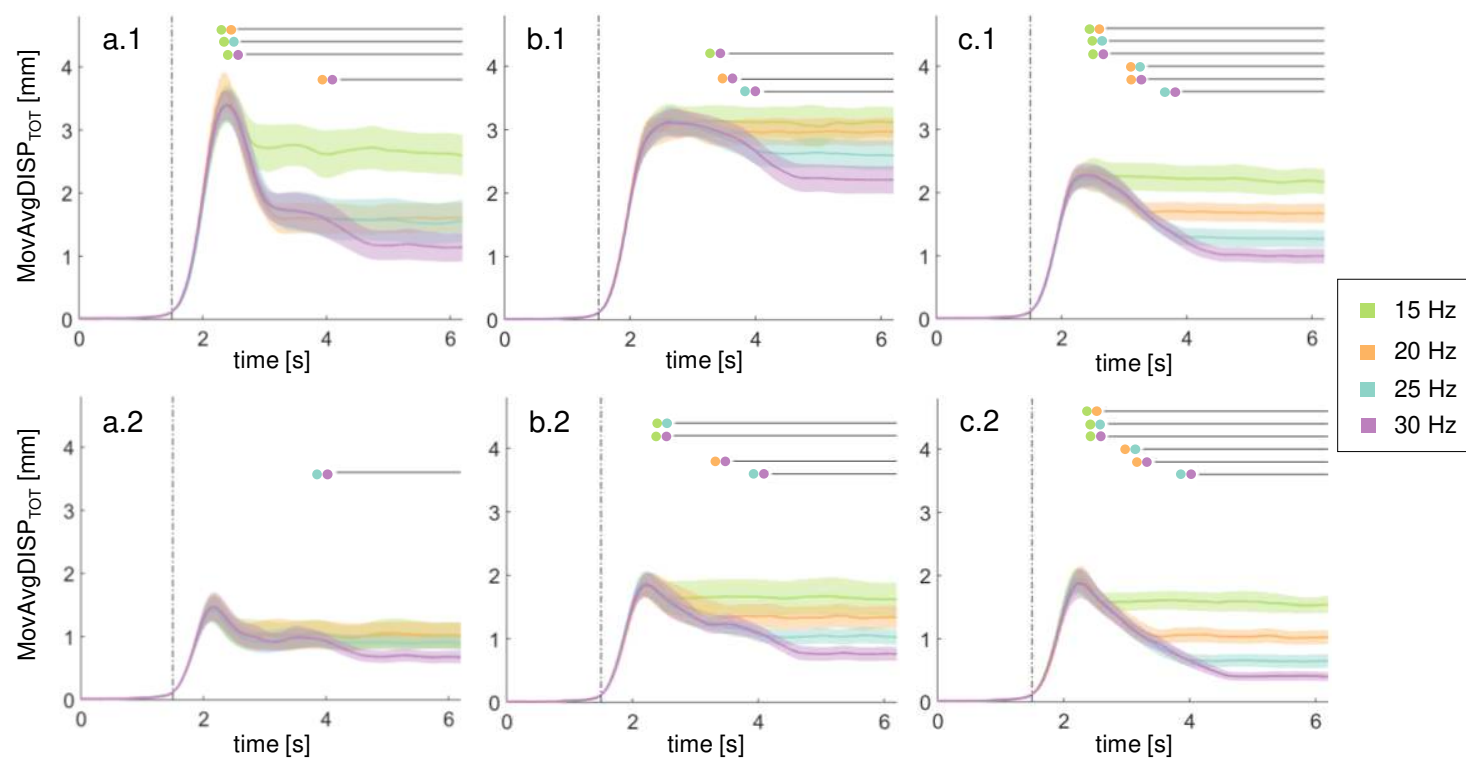

Figure 2: Muscle dynamics during WBVs at different frequencies and postures. Moving average of the total displacement (mean +/- standard error) for each muscle ( $a=G L, b=S O L, c=T A)(N=25)$. The top row (.1) shows the mechanical responses while subjects underwent the WBVs in Hack Squat; the bottom row (.2) shows the responses while subjects were in Fore Feet. The results of the cluster-based permutation tests are indicated by the black lines 
$1(p<.003125)$ and the conditions considered for each comparison are listed via the colour-wise legend. The vertical 2 dotted line represents the vibration onset

3

4
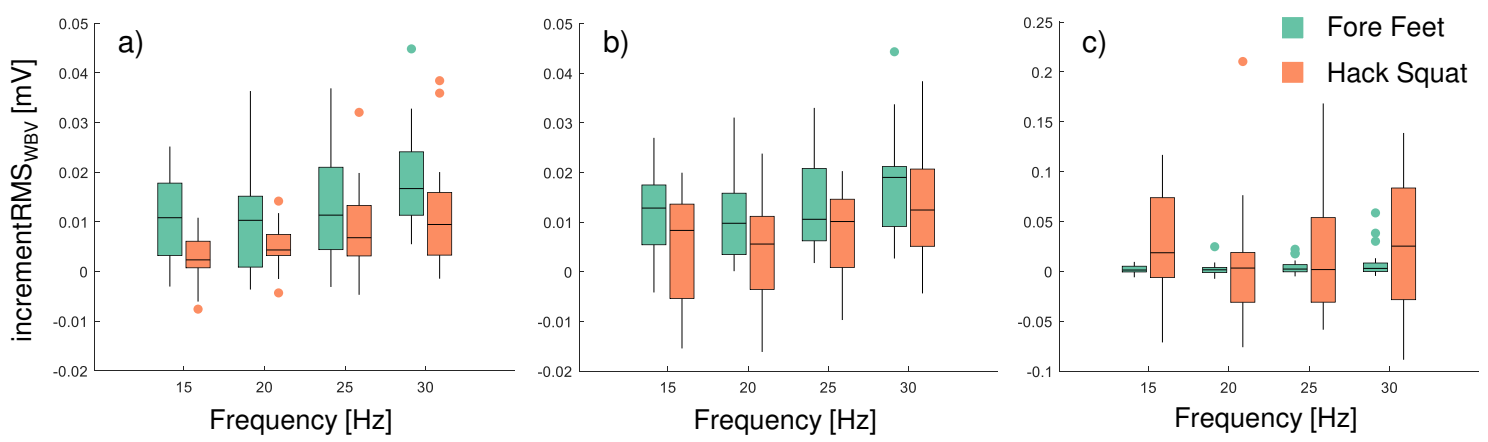

6

Figure 3: SEMG RMS ANOVA results. Box plots of incrementRMS ${ }_{W B V}$ values at different stimulation frequencies (15-

$730 \mathrm{~Hz}$ ) of $a=$ Gastrocnemius Lateralis $(N=21), b=$ Soleus $(N=21)$, and $c=$ Tibialis Anterior $(N=22)$ are shown. Different

8 colours are used to distinguish between the muscle responses in hack squat (orange) and in fore feet (dark green)

9 while the dots represent the outliers retained for the specific population. No significant interactions resulted from the

10 ANOVAs. For significant main effects of stimulation frequency and subject posture refer back to the text. The figure

11 was produced with Gramm 67 

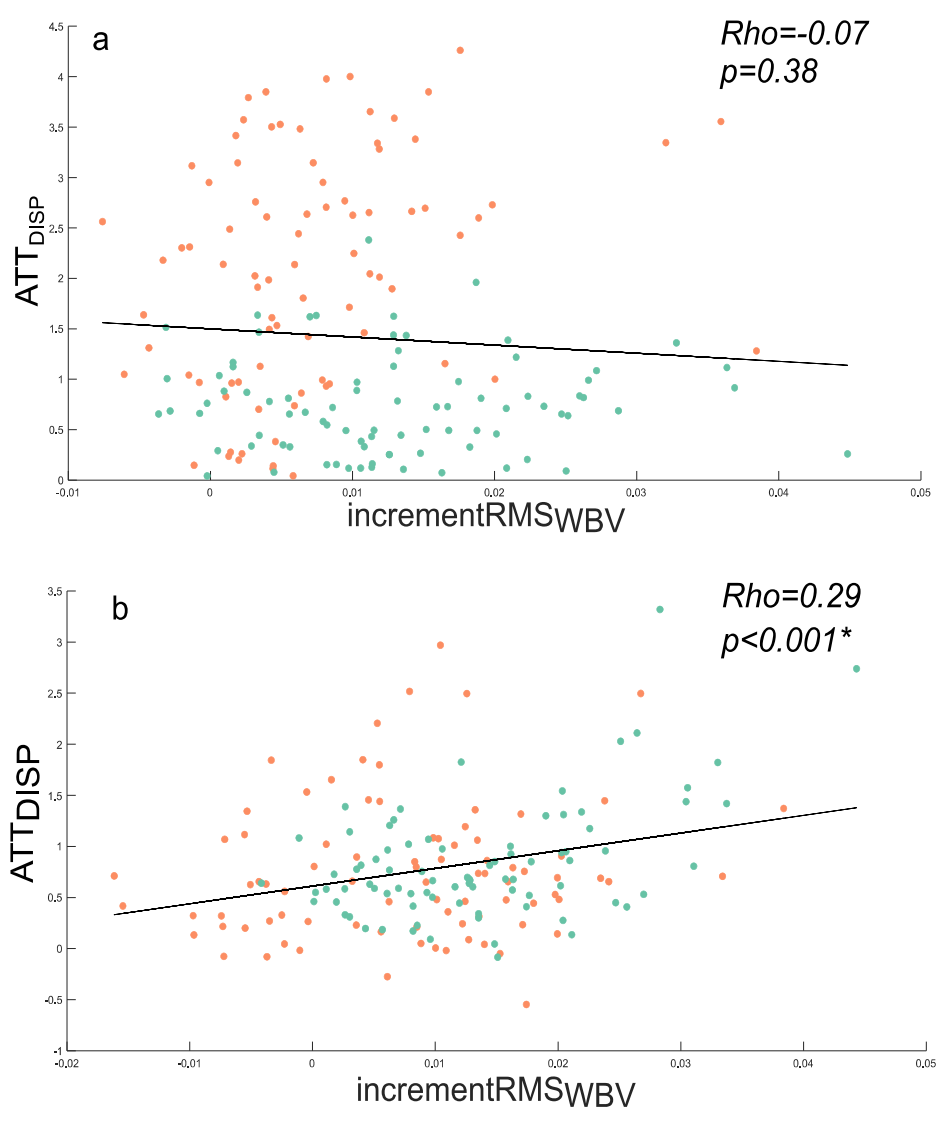

Fore Feet

Hack Squat

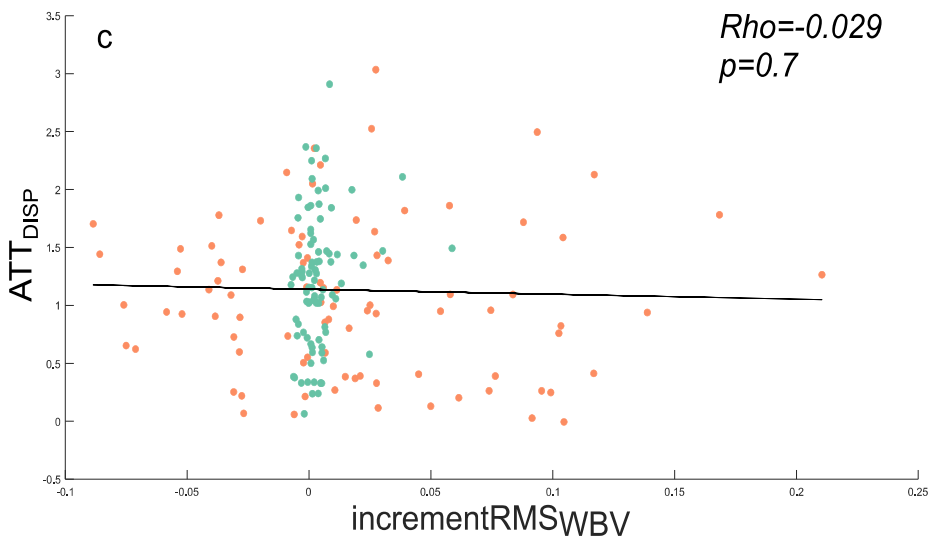

1

2

3

4

\section{Tables}

7

8

9

Figure 4: Correlation between muscle activity and displacement attenuation. Person correlation analyses were performed between the increase of activation of $G L(A), S O L(B)$ and TA (C) and their respective displacement attenuation ( $N=168$ ). The asterisk depicts the significant correlation found for the SOL muscle.

Table 1: Results of the Wilcoxon signed rank tests used to test whether the WBV-induced increment of muscle activation (increment $R M S_{W B V}$ ) was significant. The mean (SD) of increment $R M S_{W B V}$ measured in each condition is reported, as well as the $p$-value of each test $(N=25)$. The asterisk denotes statistical significance. 


\begin{tabular}{lllll|llll} 
& HS & & & & FF & & & \\
& $\mathbf{1 5 ~ H z}$ & $\mathbf{2 0 ~ H z}$ & $\mathbf{2 5 ~ H z}$ & $\mathbf{3 0 ~ H z}$ & $\mathbf{1 5 ~ H z}$ & $\mathbf{2 0 ~ H z}$ & $\mathbf{2 5 ~ H z}$ & $\mathbf{3 0 ~ H z}$ \\
\hline \multirow{2}{*}{$\mathrm{GL}$} & .0026 & .0043 & .0091 & .0160 & .0158 & .0120 & .0193 & .0329 \\
& $(.0067)$ & $(.0063)$ & $(.0077)$ & $(.0154)$ & $(.0269)$ & $(.0139)$ & $(.0298)$ & $(.0744)$ \\
& $p=.023^{*}$ & $p=.002^{*}$ & $p<.0001^{*}$ & $p<.0001^{*}$ & $p<.0001^{*}$ & $p<.0001^{*}$ & $p<.0001^{*}$ & $p<.0001^{*}$ \\
\hline SO & .0031 & .0031 & .0092 & .0179 & .0203 & .0159 & .0194 & .0325 \\
& $(.0157)$ & $(.0143)$ & $(.0186)$ & $(.0157)$ & $(.0372)$ & $(.0171)$ & $(.0217)$ & $(.0641)$ \\
L & $p=.051$ & $p=.039^{*}$ & $p=.002^{*}$ & $p<.0001^{*}$ & $p<.0001^{*}$ & $p<.0001^{*}$ & $p<.0001^{*}$ & $p<.0001^{*}$ \\
\hline \multirow{2}{*}{ TA } & .0349 & .0128 & .0229 & .0310 & .0026 & .0023 & .0044 & .0089 \\
& $(.0571)$ & $(.0622)$ & $(.0633)$ & $(.0644)$ & $(.0053)$ & $(.0080)$ & $(.0090)$ & $(.0166)$ \\
& $p=.012^{*}$ & $p=.396$ & $p=.241$ & $p=.045^{*}$ & $p=.021^{*}$ & $p=.165$ & $p=.019^{*}$ & $p=.01^{*}$ \\
\hline
\end{tabular}

1 